Manuscript date: September 21, 1999

Journal: J. Phys. Chem.

\title{
Bond dissociation energies of the tungsten fluorides and their singly-charged ions: a density functional survey
}

Kenneth G. Dyall

Thermosciences Institute, NASA Ames Research Center, Mail Stop 230-3, Moffett Field, CA 94035-1000, U.S.A. Email: dyall@pegasus.arc.nasa.gov

The dissociation of $\mathrm{WF}_{6}$ and the related singly-charged cations and anions into the lower fluorides and fluorine atoms has been investigated theoretically using density functional theory (B3LYP) and relativistic effective core potentials, with estimates of spin-orbit effects included using a simple model. The inclusion of spin-orbit is essential for a correct description of the thermochemistry. The total atomization energy of the neutral and anionic $\mathrm{WF}_{6}$ is reproduced to within $25 \mathrm{kcal} / \mathrm{mol}$, but comparison of individual bond dissociation energies with available experimental data shows discrepancies of up to $10 \mathrm{kcal} / \mathrm{mol}$. The results are nevertheless useful to help resolve discrepancies in experimental data and provide estimates of missing data. 


\section{Introduction}

Tungsten hexafluoride is an important species in the manufacture of semiconductor devices. It is used for deposition of tungsten metal to form interconnects, because of its high conductivity and its compatibility with the silicon substrate. In the modelling of the deposition process ${ }^{1,2}$, a knowledge of the bond dissociation energies of the various species in the gas phase, is required, as well as that of surface species. The heat of formation of $\mathrm{WF}_{6}$ is well-known ${ }^{3}$, and the heats of formation of several of the neutral tungsten fluorides were determined experimentally some time ago ${ }^{4}$, but some uncertainties in the data still remain. Moreover, $\mathrm{WF}_{6}$ has a large electron affinity, so that in a plasma environment where there are many free electrons, there is the possibility of forming both positive and negative ions. Experimental data exist for some of the negative ion species as well ${ }^{5-9}$, but again there are large uncertainities in some of the data and large disagreements between some. Little is known about the positive ions apart from a few ionization potentials ${ }^{4,10}$.

On the theoretical front, there have been few accurate studies ${ }^{11}$ of $\mathrm{WF}_{6}$, and no studies of tungsten fluorides other than $W_{6}$ until the recent study by Gutowski ${ }^{12}$ of the performance of density functional theory (DFT) for $\mathrm{WF}_{4}$. There is therefore considerable scope for a theortical study both to interpret the experimental data and to fill in some of the missing data. The reactions which will be investigated in this paper are

$$
\begin{aligned}
& \mathrm{WF}_{n} \rightarrow \mathrm{WF}_{n-1}+\mathrm{F} \\
& \mathrm{WF}_{n}^{+} \rightarrow \mathrm{WF}_{n-1}^{+}+\mathrm{F} \\
& \mathrm{WF}_{n}^{-} \rightarrow \mathrm{WF}_{n}+e^{-} \\
& \mathrm{WF}_{n} \rightarrow \mathrm{WF}_{n}^{+}+e^{-} .
\end{aligned}
$$

For the lower $n$ values in which there are several electrons in the $5 d$ shell of the metal, there are a number of possible states and spin multiplicities to be considered. If any of these are very low-lying there is a chance that they may be thermally populated, and at the elevated temperatures common in plasma processing, higher excited states may be populated as well.

The purpose of this study is to survey the thermochemistry of tungsten hexafluoride and its singly-charged cations and anions and in the process to obtain estimates of the bond dissociation energies at a modest level of accuracy. High accuracy results, of better than 
$1 \mathrm{kcal} / \mathrm{mol}$, are at present unobtainable, for the reason that it is necessary to take into account both scalar and spin-orbit relativistic effects along with electron correlation and there are no computer programs currently available that will do all three simultaneously to the desired level of accuracy. The magnitude of the problem is in fact illustrated in this paper. Instead we content ourselves with a goal of $2-4 \mathrm{kcal} / \mathrm{mol}$, which is generally achievable with density functional theory (DFT) combined with estimates of spin-orbit interaction.

\section{Methods}

Since tungsten is a heavy atom where relativistic effects are important, the calculations have been performed using the Hay/Wadt relativistic effective core potentials (RECPs) ${ }^{13}$. The supplied double zeta basis sets (LANL2DZ) were used on the tungsten atom, and the augmented correlation-consistent double zeta basis sets of Dunning ${ }^{14}$ were used for fluorine. All of the calculations were performed with the B3LYP hybrid density functional ${ }^{15,16}$. Gaussian $94^{17}$ was used for all calculations with the fine grid option. For some species such as $\mathrm{WF}_{2}$, calculations were performed with a $(100,434)$ Lebedev grid, to confirm the linearity of the ground state. Optimization of geometries was performed for species in several spin states and symmetries to obtain information on the excited states, within the constraints of what is feasible with DFT.

Spin-orbit effects were estimated using a simple model of the spin-orbit operator. It is assumed that the only orbitals which contribute are the $5 d$ orbitals, and among these, only those into which the fluoride ions do not donate. An estimate of the radial spin-orbit integral, $R=\int_{0}^{\infty} d r R_{5 d}^{2} Z / r^{3}$ was obtained by deperturbing Dirac-Hartree-Fock calculations on $\mathrm{W}^{5+} d^{1}$ and $\mathrm{W}^{4+} d^{2}$ states. Although the spin-orbit parameter is somewhat dependent on the charge state, the $\mathrm{W}$ atom in the complexes is likely to carry a substantial charge, so the error in the selection of the parameter should be well within the desired accuracy of the results. The value selected was $8 \mathrm{kcal} / \mathrm{mol}$. Spin-orbit effects were obtained by diagonalizing the small matrix of determinants in the space of $5 d$ orbitals discussed above, and any determinants which were considered too high in energy were deleted, though in most cases an estimate of the effect of higher-lying determinants was made.

\section{Geometries and energies}

The geometries of the neutral molecules are shown in Fig. 1, and the corresponding energetic data are reported in Table 1 . For the most part, the structures are those expected 
from simple crystal field theory. $W_{6}$ is octahedral. $W_{5} \mathrm{Jahn}_{5}$-Teller distorts from the trigonal bipyramidal $\left(D_{3 h}\right)$ geometry because the unpaired electron is in an $e^{\prime \prime}$ orbital. The lowest two states therefore have ${ }^{2} B_{2}$ and ${ }^{2} A_{2} C_{2 v}$ symmetry. This means that both of them will be populated thermally, and the ground state should essentially be considered to have a degeneracy of 4 . The ground state of $\mathrm{WF}_{4}$ is the square planar $D_{4 h}{ }^{1} A_{1 g}$ state, though the calculations give a small out-of-plane $D_{2 d}$ distortion. This is likely to be a feature of the use of DFT. There are two triplet states both of which are approximately 11 $\mathrm{kcal} / \mathrm{mol}$ higher in energy. One is tetrahedral, with the two unpaired electrons occupying the $e$ orbital and coupling to give a ${ }^{3} A_{2}$ state. The other arises from the square planar $\left(a_{1 g}\right)^{1}\left(e_{g}\right)^{1}$ state which undergoes Jahn-Teller distortion to a pair of degenerate states in $D_{2 h}$ symmetry corresponding to the two possible choices of distortion mode. The state is labelled here as ${ }^{3} B_{2 g} . \mathrm{WF}_{3}$ has a quartet ground state and is planar $D_{3 h}$; the ground state is $\left(a_{1}^{\prime}\right)^{1}\left(e^{\prime \prime}\right)^{2}{ }^{4} A_{2}^{\prime}$. The $\left(a_{1}^{\prime}\right)^{2}\left(e^{\prime \prime}\right)^{1}{ }^{2} E^{\prime \prime}$ states undergo Jahn-Teller distortion to $C_{2 v}$ symmetry, forming a ${ }^{2} B_{1} /{ }^{2} A_{2}$ pair at about 6-7 kcal $/ \mathrm{mol}$ higher than the ground state. $W F_{2}$ forms $s d_{x}$ hybrids to bond with the $\mathrm{F}$ atoms. The ground state is the linear $\left(\sigma_{g}\right)^{2}\left(\delta_{g}\right)^{2}{ }^{3} \Sigma_{g}^{-}$state; the $\left(\sigma_{g}\right)$ orbital is the equatorial $s-d_{z}$ hybrid. The lowest quintet is the $\left(\pi_{g}\right)^{2}\left(\delta_{g}\right)^{2}{ }^{5} \Sigma_{g}^{+}$state at about $10 \mathrm{kcal} / \mathrm{mol} . W F$ has a $(\sigma)^{1}(\pi)^{2}(\delta)^{2}{ }^{6} \Sigma^{+}$ground state; again the sigma orbitals form $s d_{z}$ hybrids. The lowest quartet is the $(\sigma)^{2}(\pi)^{1}(\delta)^{2}{ }^{4} \Pi$ at about $8 \mathrm{kcal} / \mathrm{mol}$.

The cation geometries are shown in Fig. 2, with the corresponding energetic data in Table 2. The HOMO for $\mathrm{WF}_{6}$ is a $t_{1 g}$ orbital which is composed of $\mathrm{F} 2 p$ orbitals. The cation undergoes Jahn-Teller distortion to three equivalent states with $D_{\mathbf{4}}$ symmetry, here designated ${ }^{2} B_{2 g}$. WF 5 loses the electron which would have been in the $e^{\prime \prime}$ orbital in $D_{3 h}$ symmetry, and therefore the cation has the expected trigonal bipyramidal geometry with $a^{1} A_{1}^{\prime}$ ground state. For $W_{4}$, loss of an electron from the $a_{1 g}$ orbital of the ground state leaves a square planar ${ }^{2} A_{1 g}$ ion state. However, this state collapses to a $D_{2 d}{ }^{2} A_{1}$ state - as do optimizations starting from a $C_{2 v}$ and a $T_{d}$ geometry. There is also a ${ }^{2} B_{2 g}$ state in $D_{2 h}$ symmetry some $70 \mathrm{kcal} / \mathrm{mol}$ higher. Ionization of $\mathrm{WF}_{3}$ leads to several potential states. From the doublet, the lowest state is the planar $D_{3 h}{ }^{1} A_{1}^{\prime}$ state with a doubly-occupied $a_{1}^{\prime}$ orbital. From the quartet, removal of the $a_{1}^{\prime}$ electron gives a planar triplet $\left(e^{\prime \prime}\right)^{2}{ }^{3} A_{2}^{\prime}$ state. Removal of an $e^{\prime \prime}$ electron from the quartet or an $a_{1}^{\prime}$ electron from the doublet leads to a $\left(a_{1}^{\prime}\right)^{1}\left(e^{\prime \prime}\right)^{1}{ }^{3} E^{\prime \prime}$ state which undergoes Jahn-Teller distortion to $C_{2 v}$ symmetry. It is this last state which is the ground state, but the other two states are only a few $\mathrm{kcal} / \mathrm{mol}$ higher in energy and must be taken into consideration. Ionization from the ground state of $\mathrm{WF}_{2}$ generates a $\left(\sigma_{g}\right)^{2}\left(\delta_{g}\right)^{1}{ }^{2} \Delta_{g}$ state and a $\left(\sigma_{g}\right)^{1}\left(\delta_{g}\right)^{2}{ }^{4} \Sigma_{g}^{-}$linear state. The latter of these is the ground state. The ${ }^{2} \Delta_{g}$ is higher in energy by more than $20 \mathrm{kcal} / \mathrm{mol}$, as are the ${ }^{4} \Pi_{g}$ and the bent doublet and quartet states. The ground state of $\mathrm{WF}^{+}$is the $(\sigma)^{1}(\pi)^{1}(\delta)^{2}{ }^{5} \Pi$ 
state. The lowest-lying excited states are the $(\sigma)^{1}(\pi)^{2}(\delta)^{1}{ }^{5} \Delta$ state and the $(\sigma)^{2}(\delta)^{2}{ }^{3} \Sigma_{g}^{-}$ state.

The anion geometries are shown in Fig. 3, with energetic data in Table 3. The LUMO for $\mathrm{WF}_{6}$ is a $t_{2 g}$ orbital. The anion therefore undergoes Jahn-Teller distortion to $D_{4 h}$ symmetry with a ${ }^{2} B_{2 g}$ ground state in a similar fashion to the cation. In $\mathrm{WF}_{5}$, the extra electron goes in the $e^{\prime \prime}$ orbital to produce a ${ }^{3} A_{2}^{\prime}$ state which retains $D_{3 h}$ symmetry. There are no other low-lying states. The LUMO in the square planar ground state of $\mathrm{WF}_{4}$ is an $e_{g}$ orbital. The molecule undergoes Jahn-Teller distortion when the extra electron is placed in it, to generate a $D_{2 h}{ }^{2} B_{2 g}$ state. Addition of the extra electron to the $e_{g}$ orbital in the Jahn-Teller distorted triplet state of the neutral restores $D_{4}$ symmetry and generates an $\left(a_{1 g}\right)^{1}\left(e_{g}\right)^{2}{ }^{4} A_{2 g}$ state. This state is very close in energy to the doublet and it is not possible to determine which of these is the ground state at this level of theory. In $\mathrm{WF}_{3}$ the extra electron goes into the $a_{1}^{\prime}$ orbital to produce an $\left(a_{1}^{\prime}\right)^{2}\left(e^{\prime \prime}\right)^{2}{ }^{3} A_{2}^{\prime}$ ground state - no other orbital occupation generates a state which is close in energy. $\mathrm{WF}_{2}^{-}$has a linear quartet ground state, with the extra electron going into the unoccupied $d \pi$ orbital to produce a $\left(\sigma_{g}\right)^{2}\left(\pi_{g}\right)^{1}\left(\delta_{g}\right)^{2}{ }^{4} \Pi_{g}$ state. The lowest excited states are the $\left(\sigma_{g}\right)^{2}\left(\delta_{g}\right)^{3}{ }^{2} \Delta_{g}$ state at about $7 \mathrm{kcal} / \mathrm{mol}$, and the $\left(\sigma_{g}\right)^{1}\left(\pi_{g}\right)^{2}\left(\delta_{g}\right)^{2}{ }^{6} \Sigma_{g}^{+}$state at about $18 \mathrm{kcal} / \mathrm{mol}$. The bent states all collapse to linear. WF- has a $(\sigma)^{2}(\pi)^{2}(\delta)^{2}{ }^{5} \Sigma^{+}$ground state. The septet corresponds to a $\sigma \rightarrow \sigma$ excitation.

\section{Spin-orbit effects}

Due to the large value of the spin-orbit splitting parameter relative to the excitation enerfges of the low-lying states in some of the species, corrections are needed in several cases. These will be discussed in the same order as in the previous subsection. The values of the spin-orbit corrections are given in Table 4.

$\mathrm{WF}_{6}$ needs no correction since it is a closed-shell species and has no occupied $5 d$ orbitals. $\mathrm{WF}_{5}$ requires only a simple diagonal shift of $-\frac{1}{2} R=-4 \mathrm{kcal} / \mathrm{mol}$. This is applied to the degenerate ${ }^{2} E^{\prime \prime}$ state, which places it below the Jahn-Teller distorted states by $2.5 \mathrm{kcal} / \mathrm{mol}$. Obviously there will be an interaction between the Jahn-Teller components as well, so the potential energy surface in the distortion mode will be extremely flat. The ground state with spin-orbit interaction is expected to be at the symmetric point. For $W_{4}$ the $A_{g}$ component of the $\left(a_{g}\right)^{1}\left(e_{g}\right)^{1}{ }^{3} E_{g}$ state has a diagonal shift of $-\frac{1}{2} R$, bringing it close to the ground state. A simple $2 \times 2$ matrix diagonalization with the planar $\left(a_{g}\right)^{2}{ }^{1} A_{g}$ state gives a ground state shift of $-10.1 \mathrm{kcal} / \mathrm{mol}$. Adding a perturbative estimate of the shift of the ${ }^{3} E_{g}$ energy due 
to the $\left(e_{g}\right)^{2}{ }^{3} A_{2 g}$ gives a further shift of $-0.7 \mathrm{kcal} / \mathrm{mol}$. Both the ${ }^{4} A_{2}^{\prime}$ and ${ }^{2} E^{\prime \prime}$ states of $\mathrm{WF}_{3}$ split under the double group into $E_{1 / 2}$ and $E_{3 / 2}$ states. A similar treatment as for $\mathrm{WF}_{4}$ with these two states and a perturbative estimate for higher states gives a ground state shift of $-4.8 \mathrm{kcal} / \mathrm{mol}$ for the $E_{1 / 2}$ component and $-5.5 \mathrm{kcal} / \mathrm{mol}$ for the $E_{3 / 2}$ component, both of which will be thermally populated. In $\mathrm{WF}_{2}$ and WF, the $\sigma$ orbitals are essentially $5 d_{z}-6 s$ hybrids, and the spin-orbit matrix elements involving these orbitals will be reduced. We have chosen a reduction of $20 \%$. The ground state of $\mathrm{WF}_{2}$ splits into two components with labels $0_{g}^{+}$and $1_{g}$. The lowest interacting excited state is the $\left(\sigma_{g}\right)\left(\pi_{g}\right)\left(\delta_{g}\right)^{2}{ }^{5} \Pi_{g}$ state which has two components with $\omega=0$ and 1 . With the reduced spin-orbit matrix element, interaction with this state gives $-4.2 \mathrm{kcal} / \mathrm{mol}$ for the $1_{g}$ component and -3.4 for the $0_{g}^{+}$ component. Estimated interaction with the other low-lying states such as the ${ }^{3} \Pi_{g}$ is 1 $\mathrm{kcal} / \mathrm{mol}$. In WF there are two low-lying ${ }^{4} \Pi$ states with configurations $(\sigma)^{2}(\delta)^{2}(\pi)^{1}$ and $(\sigma)^{1}(\delta)^{3}(\pi)^{1}$. DFT calculations appear to $m i x$ these two, so the energy reported for the ${ }^{4} \Pi$ state is probably to be taken as the energy of the lowest state. It is then difficult to estimate the effect of the other state. With the maximum possible interaction but with a scaled $\langle\sigma \mid \pi\rangle$ spin-orbit matrix element, the ground state shift is $-8.9 \mathrm{kcal} / \mathrm{mol}$ for the $\Omega$ $=5 / 2$ component, which is the lowest component. Using various assumptions about the position of the second ${ }^{4} I I$ state, a ground state shift of between -6.3 and $-6.9 \mathrm{kcal} / \mathrm{mol}$ is obtained. It is hard to judge which is the most likely, and we select the -7.0 value as being about in the middle of the range. For $\mathrm{W}$ atom we combine the DFT calculations with the experimental data to obtain the value reported here.

For $\mathrm{WF}_{6}^{+}$the hole is essentially in the $\mathrm{F} 2 p$ orbitals, and since the spin-orbit splitting on $F$ is small, it may be neglected in the molecule. $W_{5}^{+}$is similar to $W_{6}$ in having no occupied $5 d$ orbitals, and hence has no shift. $\mathrm{WF}_{4}^{+}$has a single electron in a $d_{z^{2}}$ orbital, and has no diagonal shift. The $d$ orbitals with which it will interact via the spin-orbit interaction are the orbitals into which the $\mathrm{F}^{-}$ligands are donating, and the empty orbitals will also be mixed with $\mathrm{F}$ orbitals. Hence only a small spin-orbit shift is expected, probably about $1 \mathrm{kcal} / \mathrm{mol}$. $\mathrm{WF}_{3}^{+}$is probably the most interesting since it has three very close-lying states which have large spin-orbit matrix elements connecting them. The lowest is the Jahn-Teller distorted $\left(d_{0}\right)\left(d_{1}\right)^{3} E^{\prime \prime}$ state; the other two, the $\left(d_{+1}\right)\left(d_{-1}\right){ }^{3} A_{2}^{\prime}$ and the $\left(d_{0}\right)^{2}{ }^{1} A_{1}^{\prime}$ state are practically degenerate. Inclusion of these three states in a spin-orbit $\mathrm{CI}$ at the symmetric point on the assumption that they are degenerate produces a splitting of $19 \mathrm{kcal} / \mathrm{mol}$ between each state, and thus a ground-state lowering of $16 \mathrm{kcal} / \mathrm{mol}$. In contrast, the excited states of $\mathrm{WF}_{2}^{+}$ are relatively high-lying and have small spin-orbit matrix elements with the ground state, so that the ground state shift is estimated at $-0.5 \mathrm{kcal} / \mathrm{mol}$. The ${ }^{5} \Pi$ ground state of $\mathrm{WF}^{+}$ splits into $\Omega=3,2,1,1,0^{+}$and $0^{-}$components. Interaction with the $(\pi)^{2}(\delta)^{2}{ }^{5} \Sigma^{+}$and 
$(\sigma)^{2}(\delta)^{2}{ }^{3} \Sigma^{-}$states gives a ground state of ${ }^{5} \Pi_{1}$ at $-8.2 \mathrm{kcal} / \mathrm{mol}$ relative to the ${ }^{5} \Pi$. The next component is the ${ }^{5} \Pi_{0+}$ at $-4.9 \mathrm{kcal} / \mathrm{mol}$. The ground state of $\mathrm{W}^{+}$is the $d^{5}{ }^{6} S$ state which has no first-order spin-orbit splitting.

For $\mathrm{WF}_{6}^{-}$the extra electron is in the $t_{2 g}$ orbital which undergoes Jahn-Teller distortion. In the double group, the $t_{2 g}$ orbital splits into a doubly-degenerate $\gamma_{7}$ and a quadruplydegenerate $\gamma_{8}$ representation. The former is lower in energy, and has equal weight of the three $t_{2 g}$ orbitals so it does not Jahn-Teller distort. The spin-orbit shift is $-8 \mathrm{kcal} / \mathrm{mol}$, and given that the Jahn-Teller energy is about $2 \mathrm{kcal} / \mathrm{mol}$, the overall shift relative to the unperturbed state is $-6 \mathrm{kcal} / \mathrm{mol} . \mathrm{WF}_{5}^{-}$has no diagonal shift, and the interacting excited states will be higher in energy and have reduced matrix elements because of mixing with the F $2 p$ orbitals. The ground state energy is therefore not corrected. In $\mathrm{WF}_{4}^{-}$, both the ${ }^{4} A_{2 g}$ and ${ }^{2} E_{g}$ split into $E_{1 / 2 g}$ and $E_{3 / 2 g}$ components. The ${ }^{2} E_{g}$ is approximately $2 \mathrm{kcal} / \mathrm{mol}$ higher than the ${ }^{4} A_{2 g}$ at the symmetric point, and the lowest $E_{1 / 2 g}$ and $E_{3 / 2 g}$ states then lie at approximately -7.2 and $-6.6 \mathrm{kcal} / \mathrm{mol}$ relative to the ${ }^{4} A_{2 g}$ state. An extra shift of $-1 \mathrm{kcal} / \mathrm{mol}$ due to neglected states is added to these totals. $\mathrm{WF}_{3}^{-}$has higher lying excited states and the spin-orbit shift is therefore small, estimated by perturbation arguments to be $-1 \mathrm{kcal} / \mathrm{mol}$. For $\mathrm{WF}_{2}^{-}$the ground $\left(\sigma_{g}\right)^{2}\left(\delta_{g}\right)^{2}\left(\pi_{g}\right)^{4} \Pi$ state interacts with the $\left(\sigma_{g}\right)^{2}\left(\delta_{g}\right)^{32} \Delta$ state and the $\left(\sigma_{g}\right)^{1}\left(\delta_{g}\right)^{2}\left(\pi_{g}\right)^{2}{ }^{6} \Sigma_{g}^{+}$state. The ground state has $\Omega=5 / 2$ and lies at -7.0 $\mathrm{kcal} / \mathrm{mol}$. WF ${ }^{-}$has a $\sigma^{2} \delta^{2} \pi^{2}{ }^{5} \Sigma^{+}$ground state which has no near-lying states with which it can interact, and the spin-orbit shift is likely to be small. The ground state of $\mathrm{W}^{-}$is the $d^{5} s^{2}{ }^{6} S$ state which has no first-order spin-orbit splitting.

It is clear from these results that no account of the energetics of the tungsten fluorides can hope to be quantitatively correct without the inclusion of spin-orbit effects. The planar $d^{2}$ systems show particularly large ground state shifts, and many of the other species show shifts greater than $5 \mathrm{kcal} / \mathrm{mol}$.

\section{Ionization potentials and electron affinities}

Computed ionization potentials (IP) and electron affinities (EA) in $\mathrm{eV}$ of the various species are given in Table 5 , along with the available experimental data ${ }^{4-10,18-21}$. The larger IP of $\mathrm{WF}_{6}$ reflects the fact that the electron is coming out of the $\mathrm{F} 2 p$ orbitals, whereas for all other species the electron is removed from a W $5 d$ orbital. The photoelectron value for $\mathrm{WF}_{5}$ reported by Vovna et al. ${ }^{10}$ is similar to the $\mathrm{IP}$ of $\mathrm{WF}_{6}$, and therefore must be regarded as coming from the F $2 p$ orbitals, not the $\mathrm{W} 5 d$ orbital. The values obtained here are in general agreement with the appearance potentials reported by Hildenbrand ${ }^{4}$, and display 
the same general trend. Electron affinities determined by DFT must be viewed with a little caution: the EA of $\mathrm{W}$ atom is clearly one example where it fails. Nevertheless the EA of F and $\mathrm{WF}_{6}$ are in reasonable agreement with experiment. The EA of $\mathrm{WF}_{5}$ given by De Wall et $a l^{8}$ is probably too low to be correct, if the value for $\mathrm{WF}_{4}$ is accepted.

\section{Bond dissociation energies}

Table 6 shows the computed equilibrium bond dissociation energies (BDEs) and total atomization energies for the tungsten fluorides. Because of the failure of the DFT calculations for $\mathrm{W}^{-}$, the $\mathrm{BDE}$ of $\mathrm{WF}^{-}$was calculated using reaction $\mathrm{WF}^{-} \rightarrow \mathrm{W}+\mathrm{F}^{-}$and the result corrected with the experimental electron affinities. The size of the spin-orbit effect is shown in columns 3,6 and 9 . The $B D E s$ of $W F_{4}$ and $W F_{2}$ involve a change in the total spin and therefore must involve a spin-orbit avoided crossing which could produce a barrier in the dissociation channel. The very small value of the $\mathrm{BDE}$ for $\mathrm{WF}_{6}^{+}$can be explained in terms of the differences in IP between $W_{6}$ and $W_{5}$ as discussed above. Ionization of $\mathrm{WF}_{8}$ with only a small amount of excess energy is likely to produce fragmentation. Removal of the second and third $\mathrm{F}$ atoms from $\mathrm{WF}_{6}^{+}$is relatively easier than the remaining three, which require an extra $30 \mathrm{kcal} / \mathrm{mol}$ for each $\mathrm{F}$. In contrast, the anion $\mathrm{WF}_{6}^{-}$is particularly strongly bound. There is an alternation in the bond strengths of the $\mathrm{WF}_{n}^{-}$anions with $n$, with the even numbers having a higher BDE.

Experimental data are available for several species. For the neutral systems, an accurate value can be deduced for WF from the JANAF data ${ }^{3}$, and values for $\mathrm{WF}_{n}, n=3-6$ have been reported by Hildenbrand ${ }^{4}$. The value for $W_{2}$ can be derived from these. The theoretical data with the zero-point and thermal corrections are presented in Table 7 along with these experimental data. Since the final theoretical numbers are underestimated, a scaling factor has been introduced which adjusts the total atomization energy to the experimental value. The $B D E s$ of $W_{6}$ and $W_{5}$ are obviously too small by about $10 \mathrm{kcal} / \mathrm{mol}$, even after scaling, whereas the unscaled BDEs of $\mathrm{WF}_{4}$ and $\mathrm{WF}_{3}$ are close to the experimental values. The BDE of $\mathrm{WF}_{2}$ is somewhat too high and that of WF too low, suggesting that, if anything, the effect of spin-orbit coupling may have been underestimated. Nonetheless, even an adjustment of $5 \mathrm{kcal} / \mathrm{mol}$ would not bring the BDE of WF close enough to experiment, although it would improve the $\mathrm{BDE}$ of $\mathrm{WF}_{2}$. No such easy explanation can be found for $W_{6}$ and $W_{5}$, however. The experimental errors in the derived heats of formation are of the order of $2-3$ $\mathrm{kcal} / \mathrm{mol}$, so the differences are definitely outside the experimental errors.

For the positive ions, estimates of the experimental BDEs may be obtained by use of 
the IP data from Hildenbrand ${ }^{4}$. The values for $W_{5}, W F_{4}$ and $W_{3}$ are 103,100 and $120 \mathrm{kcal} / \mathrm{mol}$, which compare reasonably well with the theoretical values of 99,99 and 130 $\mathrm{kcal} / \mathrm{mol}$, given that the thermal and zero-point energies approximately cancel and that the errors due to the IP data are 5,7 and $12 \mathrm{kcal} / \mathrm{mol}$.

It is for the negative ions that there is more disagreement among the experimental data. The heat of formation of $\mathrm{WF}_{6}^{-}$is $-490 \pm 5 \mathrm{kcal} / \mathrm{mol}$. Using $\Delta H_{f}^{o}=-337 \mathrm{kcal} / \mathrm{mol}$ from Thynne and Harland ${ }^{7}$ for $W_{5}^{-}$and $\Delta H_{f}^{o}=-306 \mathrm{kcal} / \mathrm{mol}$ from DeWall and Neuert ${ }^{8}$ for $\mathrm{WF}_{4}^{-}$the $\mathrm{BDEs}$ of $\mathrm{WF}_{6}^{-}$and $\mathrm{WF}_{5}^{-}$are 172 and $50 \mathrm{kcal} / \mathrm{mol}$, respectively. This suggests that the Thynne and Harland heat of formation is too high, and should be adjusted downwards by perhaps $40 \mathrm{kcal} / \mathrm{mol}$, to $-377 \mathrm{kcal} / \mathrm{mol}$. The enthalpy change for the reaction $\mathrm{WF}_{6}^{-} \rightarrow$ $\mathrm{WF}_{4}^{-}+2 \mathrm{~F}$ is the sum of these two BDEs, $222 \mathrm{kcal} / \mathrm{mol}$, which is in reasonable accord with the theoretical estimate of $239 \mathrm{kcal} / \mathrm{mol}$. However, using the electron affinity for $\mathrm{WF}_{4}$ from DeWall and Neuert and the heat of formation of $\mathrm{WF}_{4}$ from Hildenbrand, a value of -286 $\mathrm{kcal} / \mathrm{mol}$ is obtained for $\Delta H_{f}^{o}\left(\mathrm{WF}_{4}^{-}\right)$. This brings the reaction energy above to $242 \mathrm{kcal} / \mathrm{mol}$ which happens to be close to the theoretical estimate. The atomization enthalpy, $\mathrm{WF}_{6}^{-} \rightarrow$ $\mathrm{W}^{-}+6 \mathrm{~F}$, is calculated using the experimental electron affinities to be $791 \mathrm{kcal} / \mathrm{mol}$. Again, the theoretical numbers underestimate this value, in this case by $15 \mathrm{kcal} / \mathrm{mol}$.

\section{Conclusions}

The bond dissociation energies of the neutral and singly-charged anionic and cationic tungsten fluorides have been calculated using density functional theory with the B3LYP functional and the Hay/Wadt relativistic effective core potential for W, with an estimate of spin-orbit effects. Spin-orbit effects are large for some molecules and cannot be neglected if accurate thermochemistry is required. Density functional theory performs moderately well, but the maximum errors are disappointingly large, at around $10 \mathrm{kcal} / \mathrm{mol}$. It is possible but not likely that changes in the basis set, the functional or the core potential will dramatically improve these figures.

Acknowledgements. The author was supported by NASA Contract No. NAS2-14031. All calculations were performed on the Computational Chemistry Branch IBM RS/6000 workstation cluster. 


\section{References}

(1) R. Arora, and R. Pollard, J. Electrochem. Soc. 1991, 198, 1523.

(2) K. J. Kuijlaars, C. R. Kleijn, and H. E. A. van den Akker, Thin Solid Films 1995, $270,456$.

(3) M. W. Chase Jr., J. Phys. Chem. Ref. Data 1998, 9, 1.

(4) D. L. Hildenbrand, J. Chem. Phys. 1975, 62, 3074.

(5) P. M. George, and J. L. Beauchamp, Chem. Phys. 1979, $96,345$.

(6) A. A. Viggiano, J. F. Paulson, F. Dale, M. Henchman, N. G. Adams, and D. Smith, J. Phys. Chem. 1985, 89, 2264.

(7) J. C. J. Thynne, and P. W. Harland, Int. J. Mass Spectrom. Ion Phys. 1973, 11, 137.

(8) R. DeWall, and H. Neuert, Z. Naturfor. 1977, $32 A, 1968$.

(9) H. Dispert, and K. Lacmann, Chem. Phys. Lett. 1977, 45, 311.

(10) V. I. Vovna, A. S. Dudin, L. M. Avkhutskii, S. N. Lopatin, and E. G. Ippolitov, Russ. J. Inorg. Chem. 1979, 24, 1135.

(11) A search of the Quantum Chemistry Library Database showed 14 entries for $\mathrm{WF}_{6}$ of which only a few were high-level calculations, and none on the thermochemistry..

(12) M. Gutowski, Int. J. Quantum Chem. 1999, 73, 369.

(13) W. R. Wadt, and P. J. Hay, J. Chem. Phys. 1985, 82, 270, 284, 299.

(14) T. H. Dunning, Jr., J. Chem. Phys. 1989, 90, 1007.

(15) A. D. Becke, J. Chem. Phys. 1993, 98, 5648.

(16) P. J. Stephens, F. J. Devlin, C. F. Chabalowski, and M. J. Frisch, J. Phys. Chem. 1994, 98, 11623.

(17) Gaussian 94, Revision D.1, M. J. Frisch, G. W. Trucks, H. B. Schlegel, P. M. W. Gill, B. G. Johnson, M. A. Robb, J. R. Cheeseman, T. Keith, G. A. Petersson, J. A. Montgomery, K. Raghavachari, M. A. Al-Laham, V. G. Zakrzewski, J. V. Ortiz, J. B. Foresman, J. Cioslowski, B. B. Stefanov, A. Nanayakkara, M. Challacombe, C. Y. Peng, P. Y. Ayala, W. Chen, M. W. Wong, J. L. Andres, E. S. Replogle, R. Gomperts, R. L. Martin, D. J. Fox, J. S. Binkley, D. J. Defrees, J. Baker, J. P. Stewart; M. Head-Gordon, C. Gonzalez, and J. A. Pople, Gaussian, Inc., Pittsburgh PA, 1995.. 
(18) C. E. Moore, Natl. Stand. Ref. Data Ser. 1970, $34,1$.

(19) R. N. Compton, P. W. Reinhardt, and C. D. Cooper, J. Chem. Phys. 1978, 68, 2023.

(20) A. A. Bengali, S. M. Casey, C.-L. Cheng, J. P. Dick, T. Fenn, P. W. Villaalta, and D. G. Leopold, J. Am. Chem. Soc. 1992, $114,5257$.

(21) C. Blondel, P. Cacciani, C. Delsart, and R. Trainham, Phys. Rev. A 1989, 40, 3698 . 
TABLE 1: Symmetries and energies of neutral species. Total energies are given in Hartrees, relative energies in $\mathrm{kcal} / \mathrm{mol}$.

\begin{tabular}{ccccc}
\hline Species & Molec. Symm. & State Symm. & Total energy & Relative energy \\
\hline $\mathrm{WF}_{6}$ & $O_{h}$ & ${ }^{1} A_{1 g}$ & -667.22341 & \\
$\mathrm{WF}_{3}$ & $C_{2 v}$ & ${ }^{2} B_{2}$ & -567.30258 & \\
& ${ }^{2} A_{2}$ & -567.30241 & 0.1 \\
& $D_{3 h}$ & ${ }^{2} E^{\prime \prime}$ & -567.30005 & 1.5 \\
$\mathrm{WF}_{4}$ & $D_{4 h}$ & ${ }^{1} A_{1 g}$ & -467.39710 & \\
& $D_{2 d}$ & ${ }^{1} A_{1}$ & -467.39875 & -1.0 \\
& $T_{d}$ & ${ }^{3} A_{2}$ & -467.38128 & 9.9 \\
& $D_{4 h}$ & ${ }^{3} A_{2 g}$ & -467.31667 & 50.5 \\
$\mathrm{WF}_{3}$ & $D_{2 h}$ & ${ }^{3} B_{2 g}$ & -467.37725 & 12.5 \\
& $D_{3 h}$ & ${ }^{4} A_{2}^{\prime}$ & -367.47116 & \\
& $C_{2 v}$ & ${ }^{2} B_{1}$ & -367.46054 & 6.7 \\
& ${ }^{2} A_{2}$ & -367.46098 & 6.4 \\
$\mathrm{WF}_{2}$ & $D_{3 h}$ & ${ }^{2} E^{\prime \prime}$ & -367.45570 & 9.7 \\
& $D_{\infty h}$ & ${ }^{3} \Sigma_{g}^{-}$ & -267.53443 & \\
$\mathrm{WF}^{5}$ & $C_{\infty v} \Pi_{g}^{+}$ & -267.51943 & 9.4 \\
$\mathrm{~W}$ & ${ }^{6} \Sigma^{+}$ & -167.56991 & \\
& $K_{h}$ & ${ }^{4} \Pi$ & -167.55719 & 8.0 \\
$\mathrm{~F}$ & ${ }^{7} S$ & -67.63895 & \\
\hline
\end{tabular}


TABLE 2: Symmetries and energetics of positive species

\begin{tabular}{ccccc}
\hline Species & Molec. Symm. & State Symm. & Total energy & Relative energy \\
\hline $\mathrm{WF}_{6}^{+}$ & $D_{4 h}$ & ${ }^{2} A_{2 g}$ & -666.67525 & \\
$\mathrm{WF}_{5}^{+}$ & $D_{3 h}$ & ${ }^{1} A_{1}^{\prime}$ & -566.96062 & \\
$\mathrm{WF}_{4}^{+}$ & $D_{2 d}$ & ${ }^{2} A_{1}$ & -467.06173 & \\
& $D_{4 h}$ & ${ }^{2} A_{1 g}$ & -467.02256 & 24.6 \\
& $D_{2 h}$ & ${ }^{2} B_{2 g}$ & -466.95021 & 73.4 \\
$\mathrm{WF}_{3}^{+}$ & $C_{2 v}$ & ${ }^{3} A_{2}$ & -367.14035 & \\
& & ${ }^{3} B_{1}$ & -367.14030 & 0.03 \\
& $D_{3 h}$ & ${ }^{1} A_{1}^{\prime}$ & -367.13578 & 2.9 \\
& & ${ }^{3} A_{2}^{\prime}$ & -367.13587 & 2.8 \\
$\mathrm{WF}_{2}^{+}$ & $D_{\infty h}$ & ${ }^{4} \Sigma_{g}^{-}$ & -267.21812 & \\
& & ${ }^{2} \Sigma_{g}^{+}$ & -267.17322 & 28.2 \\
& & ${ }^{4} \Pi_{g}$ & -267.15743 & 38.1 \\
& ${ }^{2} \Delta_{g}$ & -267.15026 & 42.6 \\
& $C_{2 v}$ & ${ }^{4} B_{2}$ & -267.18176 & 22.8 \\
& & ${ }^{4} A_{2}$ & -267.16661 & 32.3 \\
& & ${ }^{2} A_{1}$ & -267.16733 & 31.9 \\
$\mathrm{WF}^{+}$ & ${ }^{5} \Pi$ & -167.26521 & \\
& $C_{\infty v}$ & ${ }^{5} \Sigma^{+}$ & -167.23463 & 19.2 \\
& & ${ }^{3} \Sigma^{-}$ & -167.22374 & 26.0 \\
& & ${ }^{6} S$ & -67.32594 & \\
\hline & & & & \\
& & & &
\end{tabular}


TABLE 3: Symmetries and energetics of negative species

\begin{tabular}{ccccc}
\hline Species & Molec. Symm. & State Symm. & Total energy & Relative energy \\
\hline $\mathrm{WF}_{6}^{-}$ & $D_{4 h}$ & ${ }^{2} B_{2 g}$ & -667.34116 & \\
$\mathrm{WF}_{5}^{-}$ & $D_{3 h}$ & ${ }^{3} A_{2}^{\prime}$ & -567.39544 & \\
$\mathrm{WF}_{4}^{-}$ & $D_{2 h}$ & ${ }^{2} B_{2 g}$ & -467.47984 & \\
& $D_{4 h}$ & ${ }^{4} A_{2 g}$ & -467.47718 & 1.7 \\
$\mathrm{WF}_{3}^{-}$ & $D_{3 h}$ & ${ }^{3} A_{2}^{\prime}$ & -367.52110 & \\
$\mathrm{WF}_{2}^{-}$ & $D_{\infty h}$ & ${ }^{4} \Pi_{g}$ & -267.58494 & \\
& & ${ }^{2} \Delta_{g}$ & -267.57291 & 7.5 \\
$\mathrm{WF}^{-}$ & $C_{\infty v}$ & ${ }^{6} \Sigma_{g}^{+}$ & -267.55662 & 17.8 \\
& & ${ }^{5} \Sigma^{+}$ & -167.61702 & \\
$\mathrm{~F}^{-}$ & $K_{h}$ & ${ }^{7} \Sigma^{+}$ & -167.58217 & 21.9 \\
\hline
\end{tabular}


TABLE 4: Spin-orbit corrections to ground state energies in $\mathrm{kcal} / \mathrm{mol}$. These figures include geometric changes due to Jahn-Teller distortion.

\begin{tabular}{crrr}
\hline Species & Neutral & Cation & Anion \\
\hline $\mathrm{WF}_{6}$ & 0.0 & 0.0 & -6.0 \\
$\mathrm{WF}_{3}$ & -2.5 & 0.0 & 0.0 \\
$\mathrm{WF}_{4}$ & -10.8 & -1.0 & -6.5 \\
$\mathrm{WF}_{3}$ & -5.5 & -16.1 & -1.0 \\
$\mathrm{WF}_{2}$ & -5.2 & -0.5 & -7.0 \\
$\mathrm{WF}$ & -7.0 & -8.2 & -2.0 \\
$\mathrm{~W}$ & -13.2 & 0.0 & 0.0 \\
$\mathrm{~F}$ & -0.4 & & 0.0 \\
\hline
\end{tabular}


TABLE 5: Ionization potentials and electron affinities in $\mathrm{eV}$. The columns labelled IP and EA give the property without spin-orbit effects; the columns labelled +SO gives the property with spin-orbit effects included.

\begin{tabular}{lrrcrrl}
\hline Species & \multicolumn{1}{c}{ IP } & \multicolumn{1}{c}{ +SO } & \multicolumn{1}{c}{ Expt $^{a}$} & EA & +SO & Expt \\
\hline $\mathrm{WF}_{6}$ & 14.9 & 14.9 & & 3.2 & 3.5 & $3.5(1)^{d}, 3.4(2)^{e}, 3.7(2)^{f}$ \\
$\mathrm{WF}_{5}$ & 9.3 & 9.4 & $10.0(1), 14.9^{b}$ & 2.5 & 2.4 & $<3.5^{e},>1.8(3)^{g}, 1.25^{h}$ \\
$\mathrm{WF}_{4}$ & 9.3 & 9.7 & $9.9(1)$ & 2.1 & 1.9 & $2.6^{h},>2.3(1)^{i}$ \\
$\mathrm{WF}_{3}$ & 9.0 & 8.5 & $9.0(2)$ & 1.4 & 1.2 & \\
$\mathrm{WF}_{2}$ & 8.6 & 8.8 & $9.0(3)$ & 1.4 & 1.5 & \\
$\mathrm{WF}$ & 8.3 & 8.2 & $8.5(10)$ & 1.3 & 1.1 & \\
$\mathrm{~W}$ & 8.5 & 9.1 & $7.98^{c}$ & negative & negative & $0.817(4)^{j}$ \\
$\mathrm{~F}$ & & & & 3.56 & 3.54 & $3.4012^{k}$ \\
\hline
\end{tabular}
${ }^{a}$ Ref. 4.
${ }^{b}$ Ref. 10.
${ }^{c}$ Ref. 18.
${ }^{h}$ Ref. 8.
${ }^{i}$ Ref. 7.
${ }^{d}$ Ref. 6.
e Ref. 5
${ }^{f}$ Ref. 9.
${ }^{g}$ Ref. 19.
j Ref. 20 .
${ }^{k}$ Ref. 21. 
TABLE 6: Equilibrium bond dissociation energies in kcal $/ \mathrm{mol}$. Columns labelled SF give the spin-free value, columns labelled SO give the spin-orbit correction.

\begin{tabular}{lccrrrrrrr}
\hline & \multicolumn{3}{c}{ Neutral } & \multicolumn{3}{c}{ Cation } & \multicolumn{3}{c}{ Anion } \\
Species & SF & SO & Total & \multicolumn{1}{c}{ SF } & \multicolumn{1}{c}{ SO } & Total & SF & SO & Total \\
\hline $\mathrm{WF}_{6}$ & 113.8 & -2.9 & 110.9 & 15.6 & -0.4 & 15.2 & 129.4 & +5.6 & 135.0 \\
$\mathrm{WF}_{5}$ & 103.1 & -8.7 & 94.4 & 100.0 & -1.4 & 98.6 & 110.5 & -6.9 & 103.6 \\
$\mathrm{WF}_{4}$ & 118.1 & +4.9 & 123.0 & 114.1 & -15.4 & 98.7 & 137.6 & +5.1 & 142.7 \\
$\mathrm{WF}_{3}$ & 123.6 & -0.1 & 123.5 & 114.7 & +15.0 & 129.7 & 123.4 & -4.4 & 117.0 \\
$\mathrm{WF}_{2}$ & 141.2 & -2.2 & 139.0 & 133.9 & -8.1 & 125.8 & 143.3 & +4.6 & 147.9 \\
$\mathrm{WF}$ & 125.0 & -6.6 & 118.4 & 125.4 & +7.8 & 133.2 & 128.3 & +1.6 & 129.9 \\
Sum & 724.8 & & 709.2 & 603.7 & -2.5 & 601.2 & 772.5 & +3.6 & 776.1 \\
\hline
\end{tabular}


TABLE 7: Bond dissociation energies and enthalpies in kcal $/ \mathrm{mol}$ for $\mathrm{WF}_{n} \rightarrow$ $W_{n-1}+F$ and total atomization energy and enthalpy of $W F_{6}$. Experimental data are derived from Hildenbrand ${ }^{4}$ and the JANAF compilation ${ }^{3}$.

\begin{tabular}{crcccccl}
\hline $\mathrm{WF}_{6}$ & $\mathrm{WF}_{5}$ & $\mathrm{WF}_{4}$ & $\mathrm{WF}_{3}$ & $\mathrm{WF}_{2}$ & $\mathrm{WF}$ & atomization & \multicolumn{1}{c}{ description } \\
\hline 114 & 103 & 118 & 124 & 141 & 125 & 725 & $\Delta E_{\text {e spin free }}$ \\
111 & 94 & 123 & 124 & 139 & 118 & 709 & $\Delta E_{\mathrm{e}}$ with spin-orbit \\
109 & 93 & 121 & 122 & 138 & 118 & 701 & $\Delta E_{0}=\Delta H^{\circ}$ at $0 \mathrm{~K}$ \\
110 & 94 & 122 & 123 & 138 & 119 & 705 & $\Delta H^{\circ}$ at $298 \mathrm{~K}$ \\
112 & 96 & 125 & 126 & 142 & 121 & 723 & $\Delta H^{\circ}$ scaled at $0 \mathrm{~K}$ \\
112 & 97 & 126 & 127 & 143 & 123 & 729 & $\Delta H^{\circ}$ scaled at $298 \mathrm{~K}$ \\
121 & 106 & 120 & 120 & 132 & 130 & 729 & Experimental $\Delta H^{\circ}(298 \mathrm{~K})$ \\
\hline
\end{tabular}




\section{Figure Captions}

FIGURE 1: Geometries of neutral tungsten fluorides, with bond lengths in $\AA$ and angles in degrees.

FIGURE 2: Geometries of singly-charged tungsten fluoride cations, with bond lengths in $\AA$ and angles in degrees.

FIGURE 3: Geometries of singly-charged tungsten fluoride anions, with bond lengths in $\AA$ and angles in degrees. 

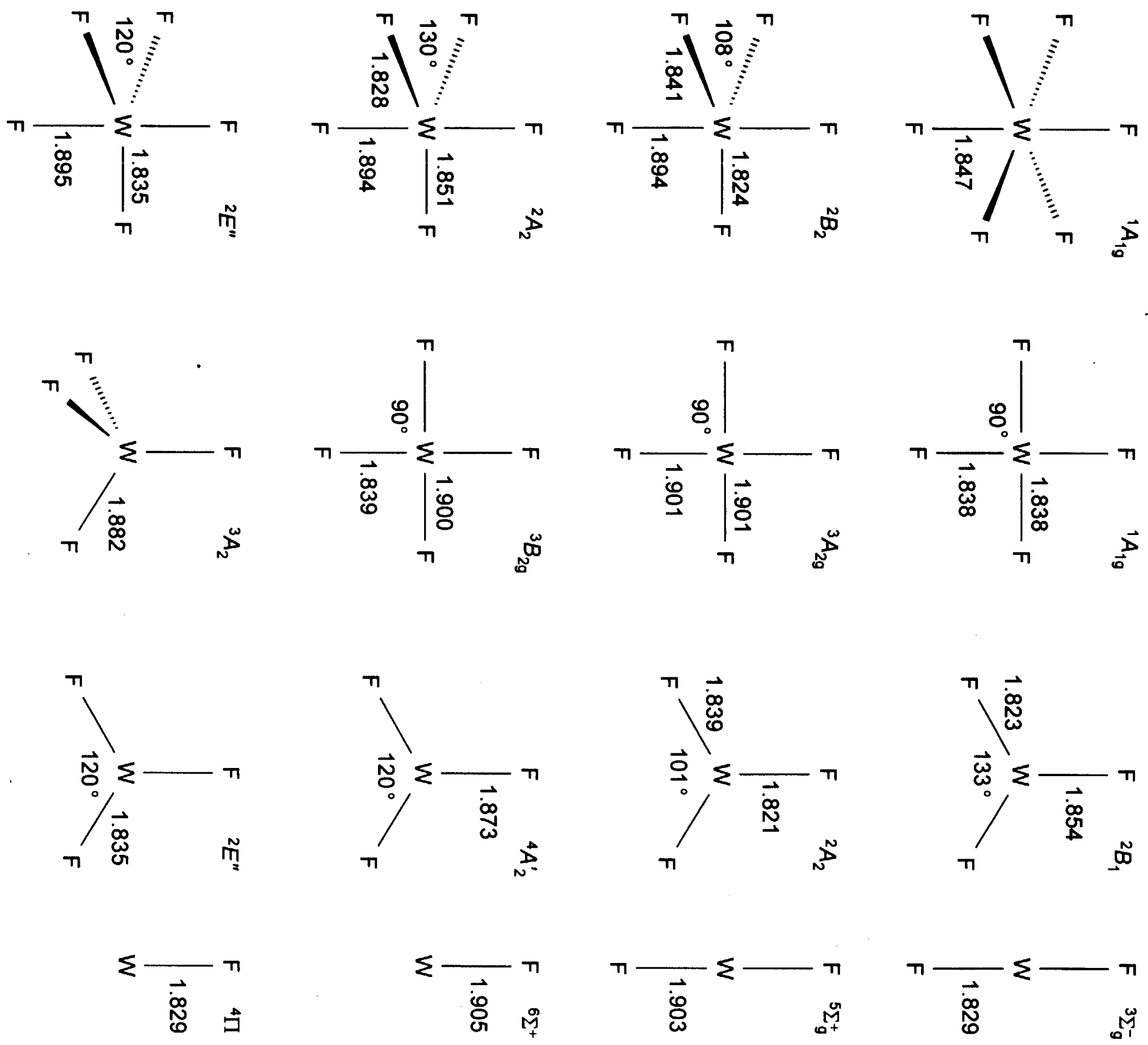

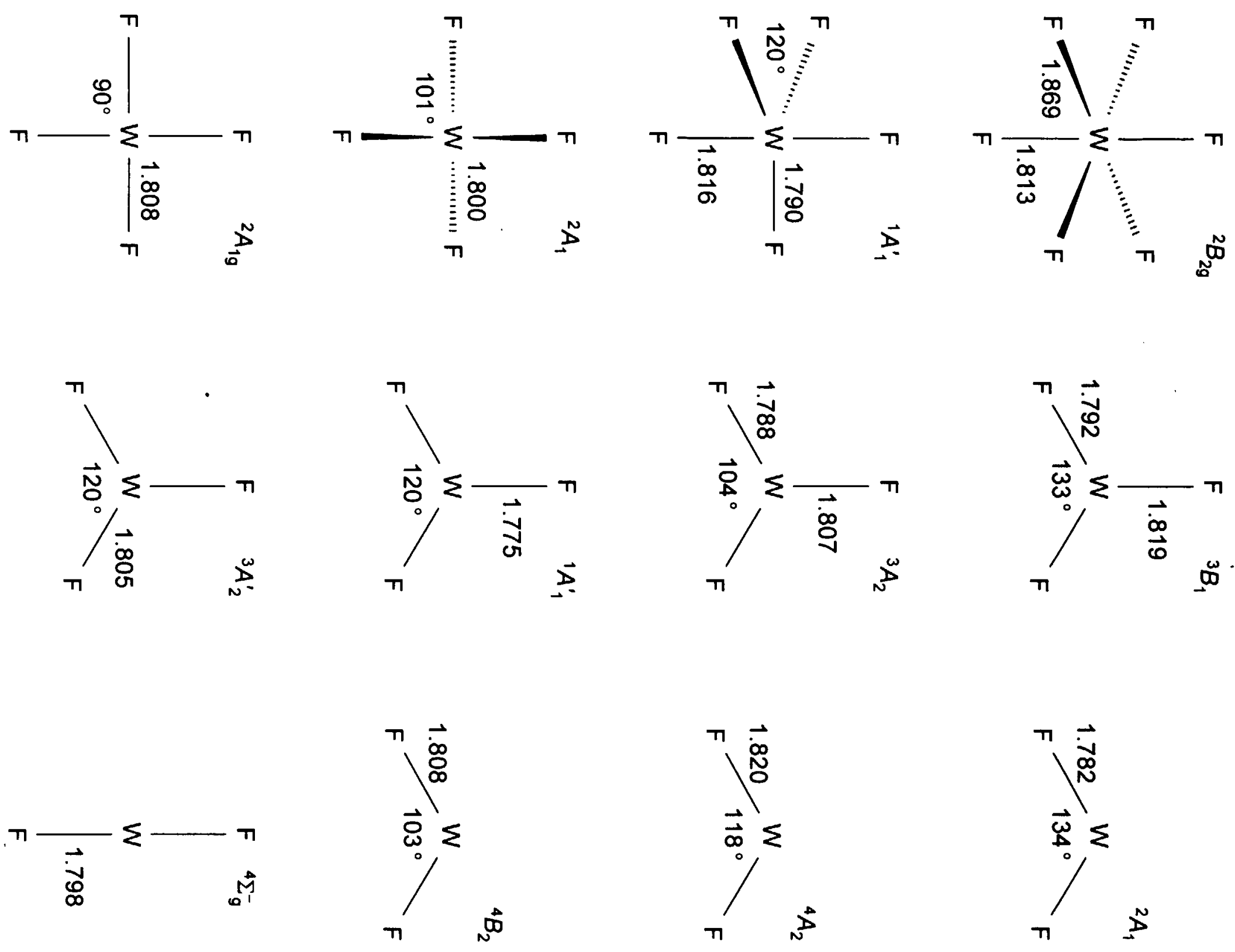

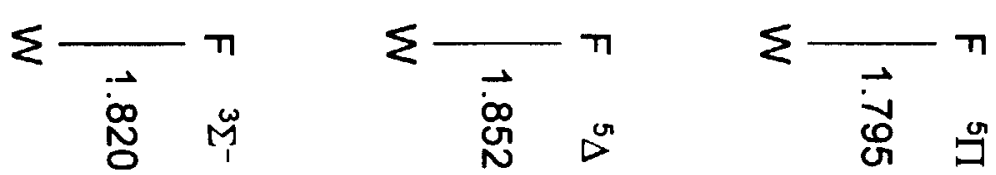
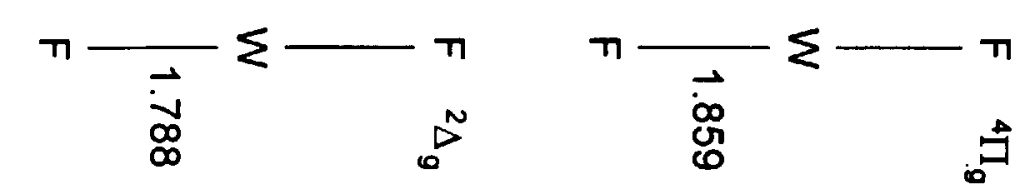

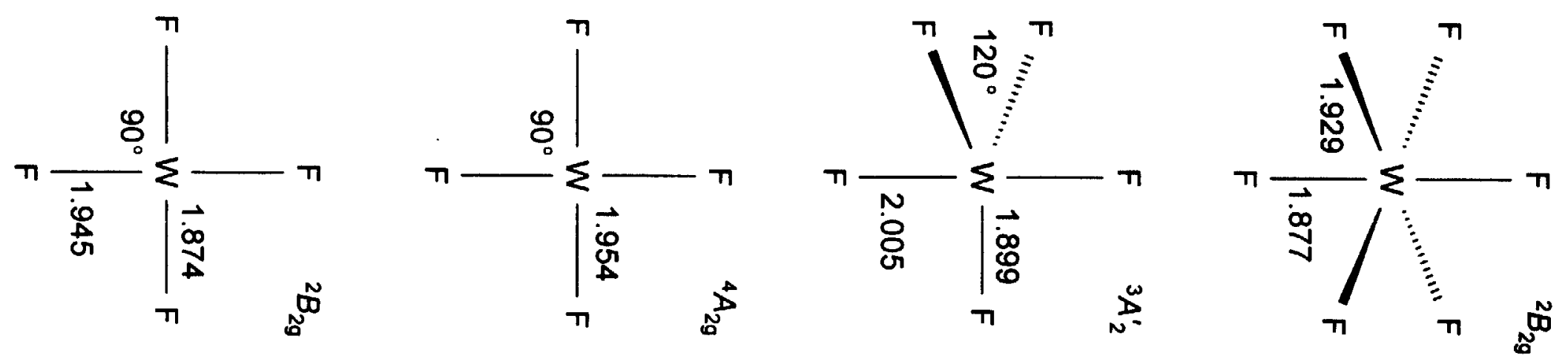

$\pi \frac{N}{\stackrel{9}{9}} \leqslant \frac{9}{0+}$
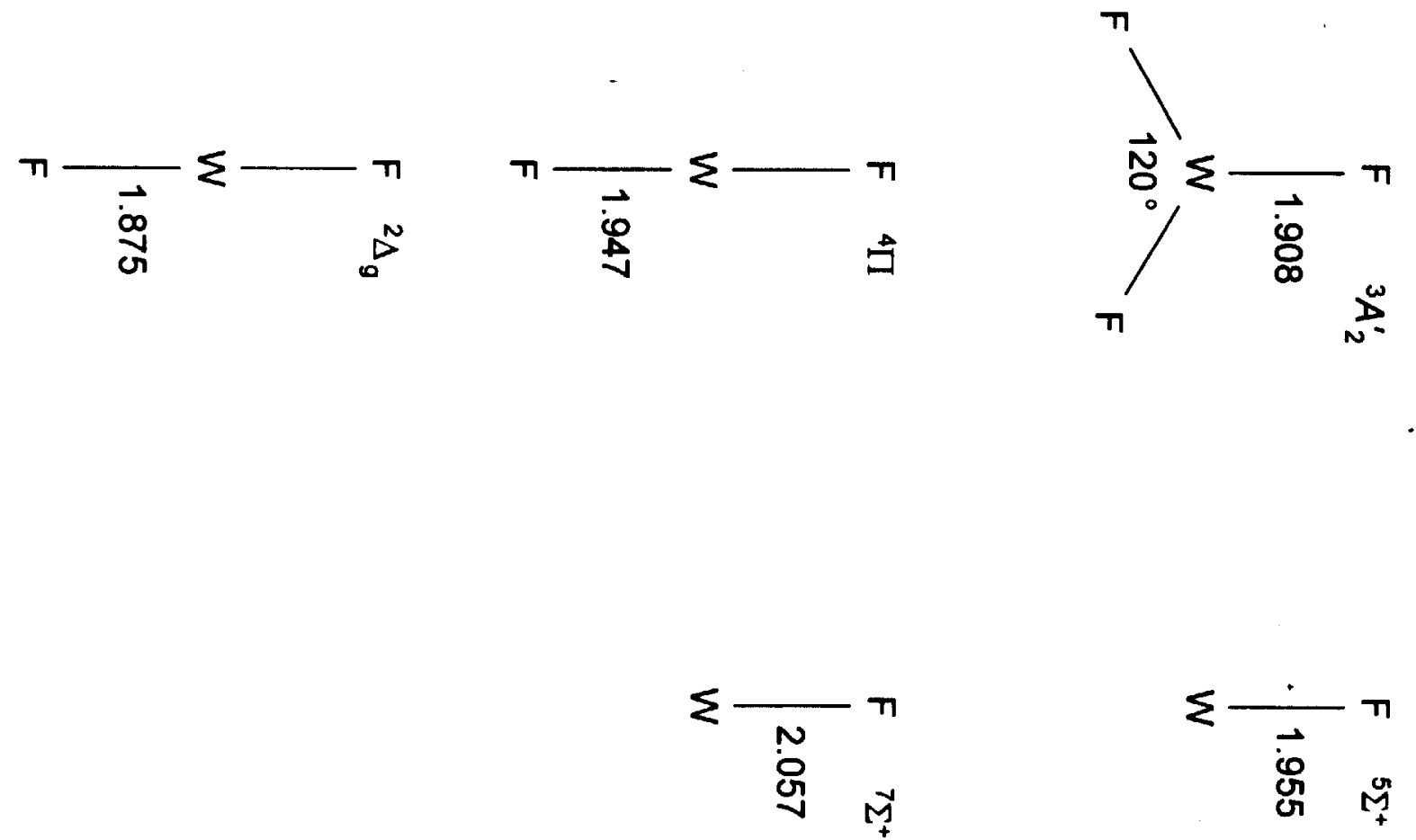


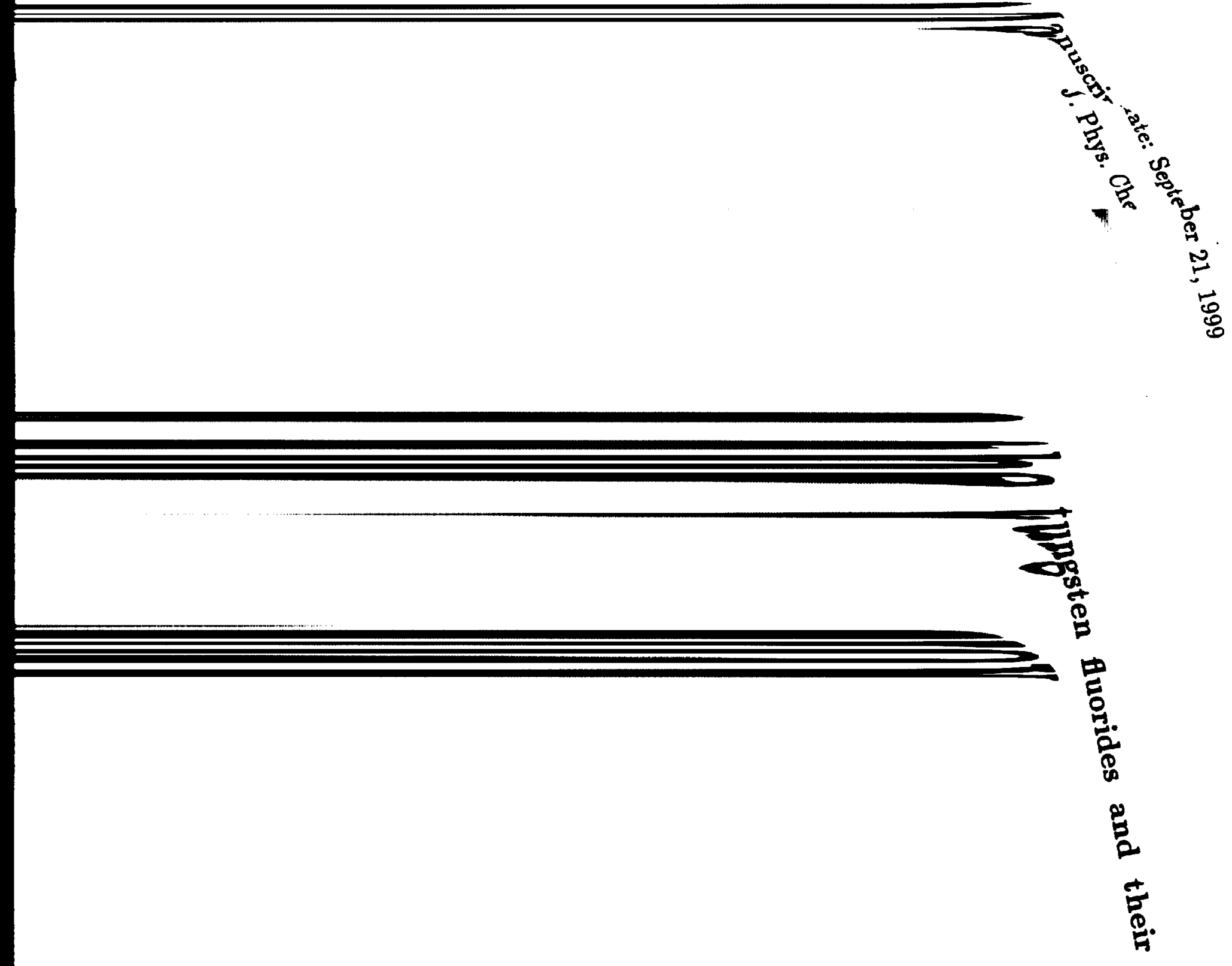




$$
]
$$

